\title{
Ultrasound Radiomics in Breast Cancer - A Literature Review
}

\author{
Yi Chen, MB ${ }^{a, b, 1}$, Xia Liu, MB ${ }^{a, 1}$, Run Wang, MD ${ }^{a}$, Xiaona Lin, MM ${ }^{a}$, Jieyu Zhong, MD ${ }^{a}$, Yan Chen, MM ${ }^{a}$, Huali \\ Cai, MB ${ }^{a}$, Yongbin Li, MM ${ }^{a}$, Zhicheng Li, MD ${ }^{c}$, Zhengming Hu, MD ${ }^{a, *}$, Desheng Sun, MD ${ }^{a, *}$ \\ ${ }^{a}$ Department of Ultrasonography, Peking University Shenzhen Hospital, Shenzhen, Guangdong, China; ${ }^{b}$ Shantou University Medical \\ College; ${ }^{c}$ Institute of Biomedical and Health Engineering, Shenzhen Institutes of Advanced Technology, Chinese Academy of Sciences, \\ Shenzhen, China
}

Received June 9, 2020; revision received July 28; accepted August 2, 2020

\begin{abstract}
As an emerging technology, radiomics has shown potential values in the field of healthcare. CT/MRI was preferred in previous radiomics researches because its images are easy to be standardized. And only until recently, an increasing number of studies focusing on the application of ultrasound radiomics in predicting molecular subtypes, identifying of malignant lesions, reactions to neoadjuvant chemotherapy, and axillary lymph node metastasis of breast cancer have been published. The purpose of this review is to summarize the steps of radiomics used in the field of breast cancer. In conclusion, ultrasound radiomics is a promising technology in diagnosing and monitoring breast cancer and further assisting physicians in patient management.
\end{abstract}

Key words: Radiomics; Ultrasonography; Breast Neoplasms

Advanced Ultrasound in Diagnosis and Therapy 2021;01:012-017

DOI: 10.37015/AUDT.2021.200052

\section{Introduction}

Breast cancer is the most common malignant tumor and one of the main causes of cancer death in women in most countries in 2018 [1]. Early diagnosis and prediction of prognosis and treatment response are essential for breast cancer patient management. Medical imaging modalities for evaluating breast cancer include magnetic resonance imaging (MRI), computed tomography (CT), positron emission tomographycomputed tomography (PET-CT), ultrasonography (US), and mammography. However, subjective and semi-quantitative analysis of images could result in some hidden information in images being ignored. The exponential growth of medical imaging in the last decade has led to the development of radiomics.

Radiomics was first proposed by Lambin et al. in 2012 [2]. It refers to extracting a large number of quantitative image features from high-throughputscreening of medical image and analyzing these features for decision support [2,3]. Compared with traditional medical imaging, radiomics is more quantitative and objective. Currently, publications about radiomics have focused on CT, MRI and PET/CT for lung, head and neck, colon and prostate cancers. The field of radiomics in breast cancer mainly based on MRI $[4,5]$. As an essential examination tool for breast imaging, ultrasound might be a useful radiomics approach for breast cancer.

The purpose of this review is to outline the steps of radiomics used in the field of breast cancer in predicting molecular subtypes, identifying of malignant lesions, reactions to neoadjuvant chemotherapy (NACT), and management of axillary lymph node (ALN) metastasis.

\section{Radiomics}

In 2012, Lambin et al. firstly introduced the concept of radiomics [2]. The hypothesis is that quantitative analysis of medical image data can capture additional information which reflects mechanisms occurring

\footnotetext{
${ }^{I}$ Yi Chen and Xia Liu contributed equally to this work.

* Corresponding Author: Desheng Sun, Department of Ultrasonography, Peking University Shenzhen Hospital, 1120 Lianhua Road, Shenzhen, Guangdong, China.e-mail: szdssun@163.com; Zhengming Hu, e-mail: hzm0682@126.com unrestricted use, distribution and reproduction in any medium provided that the original work is properly attributed.
} 
at genetic and molecular levels, and further infer phenotypes or gene-protein signatures based on the information to improve diagnostic, prognostic, and predictive accuracy. The workflow of radiomics includes: (a) acquiring high quality and standardized imaging, (b) segmenting and defining the region of interest (ROI), either automatically with an segmentation method or manually by experienced radiologists or radiation oncologists, (c) extracting quantitative imaging features from the previously segmented ROI, including tumor size, shape, intensity, texture and so on, (d) selecting the most informative traits from the extracted features according to the reproducibility and prominence on the data, and independence from other features, (e) analyzing the selected features to establish databases and predicting models $[2,6]$.

\section{Methods}

Literature search was done on PubMed and China National Knowledge Infrastructure (CKNI) websites with the following search terms: breast cancer and radiomics, radiomics and ultrasound, breast neoplasms and radiomics. Systematic reviews, review articles, metaanalyses, and randomized controlled trials (published in the last 10 years) were considered. Besides, to be more comprehensive and thorough, the references of relevant reviews were also manually retrieved to identify eligible articles.

\section{Results}

The literature search on PubMed and CKNI identified 798 articles in total. Articles were excluded because of the following reasons: (a) letters, comments, irrelevant studies, and duplicate publication; (b) articles that did not consider breast cancer or ultrasound specifically; (c) articles that focused on quantitative studies but not radiomics. At last, 15 papers were finally included for our study (Table 1). There was no formal quality assessment about the selected papers.

Table 1 The characteristics of studies on ultrasound radiomics in breast cancer included in this review

\begin{tabular}{|c|c|c|c|c|c|c|c|}
\hline First author & Year & Country & Study design & Number of patients & Average age of patients (year) & Number of radiomics features & AUC \\
\hline Liu [7] & 2017 & China & Retrospective & 104 & NA & 404 & 0.794 \\
\hline $\operatorname{Li}[8]$ & 2017 & China & Retrospective & 204 & 52.0 & 463 & 0.732 \\
\hline Zhang [12] & 2017 & China & Retrospective & 117 & NA & 364 & 0.917 \\
\hline Suo [20] & 2017 & China & Retrospective & 158 & 55.2 & 428 & NA \\
\hline Guo [9] & 2018 & China & Retrospective & 215 & NA & 463 & 0.760 \\
\hline Zhou [13] & 2018 & China & Retrospective & 205 & 35.6 & 4224 & NA \\
\hline Lee [14] & 2018 & Korea & Retrospective & 840 & 42.0 & 730 & 0.782 \\
\hline Theek [23] & 2018 & Germany & Retrospective & NA & NA & 235 & NA \\
\hline $\mathrm{Li}[10]$ & 2019 & China & Retrospective & 96 & 51.6 & 460 & $0.987 \sim 0.999$ \\
\hline Liu [11] & 2019 & China & Retrospective & 125 & NA & 220 & NA \\
\hline Luo [15] & 2019 & China & Retrospective & 315 & 44.9 & 1044 & 0.928 \\
\hline Fleury [16] & 2019 & Brazil & Retrospective & 144 & 43.6 & NA & 0.840 \\
\hline Li [19] & 2019 & China & Retrospective & 53 & 44.8 & 1044 & 0.880 \\
\hline $\mathrm{Yu}[21]$ & 2019 & China & Retrospective & 426 & NA & 96 & 0.810 \\
\hline Sun [22] & 2020 & China & Retrospective & 479 & 48.7 & 104 & 0.886 \\
\hline
\end{tabular}

NA: not available

\section{Ultrasound Radiomics in Breast Cancer}

\section{Ultrasound radiomics and molecular subtypes}

In 2017, Liu et al. demonstrated that it was feasible to predict the expression of estrogen receptor (ER) in breast cancer using ultrasound radiomics with a highest prediction accuracy of $75.96 \%$ [7]. A retrospective study published in the same year by $\mathrm{Li}$ et al. evaluated the 
value of ultrasound radiomics in predicting hormone receptor status of invasive breast carcinoma [8]. Two hundred and four cases of invasive breast carcinomas were included and were divided into hormone receptor positive group $\left(\mathrm{ER}^{+}, \mathrm{PR}^{+}, \mathrm{HER}^{-2-}\right)$ and hormone receptor negative group $\left(\mathrm{ER}^{-}, \mathrm{PR}^{-}, \mathrm{HER}^{-2-}\right)$. Radiomics features were classified into six categories: size, shape, margin, internal echoic pattern, posterior acoustic shadow, and calcification. All involved ultrasound radiomics features except for shape were found with statistically significant differences between two groups. Compared with hormone receptor negative tumors, hormone receptor positive tumors are more likely to be smaller in size and have spiculated or angular margin, hyperechoic pattern and posterior acoustic shadowing. Besides, conjoint analysis of all radiomics features can improve the predicting performance. Guo et al. proposed an automatic radiomics approach in ultrasound to evaluate the correlation between ultrasound radiomics features and biological characteristics of breast invasive ductal carcinoma (IDC) [9]. Radiomics features were sorted into six categories: shape, margin, boundary, internal echoic pattern, posterior acoustic shadow and calcification. Four features including internal echoic pattern, posterior acoustic shadow, margin, and calcification were found statistically associated with receptor status and subtypes of breast IDC, especially internal echo pattern features. High grade triple-negative IDC are more likely to have regular shape, circumscribed margin, hypo- or complex internal echoic pattern and posterior acoustic enhancement. Inversely, low grade IDC with positive hormone receptor and $\mathrm{HER}^{-2-}$ more often have irregular shape, spiculated or angular margin, hyperechoic pattern and posterior acoustic shadow. In addition, the combination of all radiomics features can achieve higher accuracy in predicting receptor status than the single feature. In 2019, Li et al. found that ultrasound radiomics features are associated with the pathological and immunohistochemical characteristics of invasive triple-negative breast cancer (TNBC) [10]. Some features can predict the expression level of Ki67 and HER ${ }^{-2-}$, and pathological grade with high performance efficacy. In the same year, Liu et al. showed that ultrasound radiomics may be able to analyze and predict characteristics of hormone receptor expression in breast cancer [11]. They filtrated and categorized ultrasound radiomics features into size, shape, margin, internal echo pattern, posterior echo pattern and calcification of tumors. The results demonstrated that only the shape character showed no statistic difference between hormone receptor positive breast cancer $\left(\mathrm{ER}^{+}, \mathrm{PR}^{+}, \mathrm{HER}^{-2-}\right)$ and hormone receptor negative breast cancer $\left(\mathrm{ER}^{-}, \mathrm{PR}^{-}, \mathrm{HER}^{-2-}\right)$.

\section{Ultrasound radiomics and malignancy}

In 2017, Zhang et al. developed a radiomics approach on sonoelastography to distinguish between benign and malignant breast tumors [12]. Three hundred and sixtyfour high-throughput features about the shape, hardness and hardness heterogeneity of breast tumors were extracted from 117 sonoelastography images, which are all significantly different between benign and malignant tumors. Seven features related to shape, intensity and contourlet texture were selected and classified based on hierarchical clustering and three-feature selection metrics. The results demonstrated that malignant tumors are stiffer with uneven distribution than benign tumors, revealing the feasibility and value of sonoelastography radiomics in breast tumor differentiation. In 2018, a segmentation-free radiomics approach on shear-wave elastography (SWE) was proposed by Zhou et al. to distinguish malignant breast tumors from benign changes [13]. Convolutional neural network $(\mathrm{CNN})$ was used in this radiomics method to automatically extract features from the recoded SWE image data. Five hundred and forty images from 205 patients were included. The results indicated that this $\mathrm{CNN}$ based radiomics method has great clinical application value in breast tumor differentiation. In 2018, a retrospective study aimed to propose an ultrasound radiomics method to discriminate TNBC and fibroadenoma [14]. They analyzed 715 cases of fibroadenoma and 186 cases of TNBC, and then extracted 730 features from the US images. They used logistic regression with a least absolute shrinkage and selection operator (LASSO) to analyze the extracted features to construct a radiomics score. The radiomics score showed significant performance improvements in distinguishing TNBC from fibroadenoma, even in Breast Imaging Reporting and Data System (BI-RADS) category 3 and 4a lesions which are assumed as benign or low suspicious of malignancy. In 2019, a nomogram combined radiomics and BI-RADS was proposed by Luo et al. to predict breast cancer in BI-RADS category 4 or 5 lesions [15]. They retrospectively analyzed 315 patients with breast lesions that could be categorized as as $4 \mathrm{~A}, 4 \mathrm{~B}, 4 \mathrm{C}$ or 5 according to the second edition of the American College of Radiology (ACR) BI-RADS US atlas. The results showed that the nomogram had better performance in discriminating between malignant and benign breast lesions than either the radiomics score or BI-RADS category. It suggested that the nomogram incorporating radiomics and BI-RADS has a potential to be applied in predicting breast cancer in BI-RADS category 4 or 5 lesions. In 2019, Fleury et al. demonstrated that BI-RADS radiomics features extracted from ultrasound images can be used to distinguish malignant breast tumors from benign with machine 
learning algorithms [16]. Ten main radiomics features were extracted from 206 ultrasound images of breast tumors based on BI-RADS lexicon. Then they used the extracted features to categorize benign or malignant lesions via five machine learning methods. The results showed that some features, especially lesions' aspect ratio and margin features, have higher performance in distinguishing benign and malignant breast lesions, and other features which have lower performance can present better potential when associated with the first ones.

\section{Ultrasound radiomics and neoadjuvant chemotherapy}

NACT is the standard treatment for locally advanced breast cancer and recently it has been used even in early breast cancer, because it can reduce tumor size and raise breast-conserving surgery rate without increasing locoregional recurrence (LRR) rate [17]. However, the pathologic complete response (pCR) rate after NACT is low [18]. Whether ultrasound radiomics can predict the response of breast cancer after NACT has been explored to select patients who are more likely to achieve pCR.

In 2019, Li et al. explored the value of ultrasound radiomics in predicting therapeutic effect of NACT in breast cancer [19]. They extracted radiomics features from ultrasound images of 54 cases of breast cancer and used logistic regression to build an influential prediction model based on the extracted features. Ultimately, 6 selected features were included in logistic regression model. The model successfully predicted the clinical response after NACT (AUC of 0.88), suggesting that ultrasound radiomics might help to forecast curative effect of NACT in breast cancer.

\section{Ultrasound radiomics and ALN metastasis}

ALN metastasis is an essential prognostic factor in breast cancer patients and is one of the most important factors in clinical treatment options. Axillary lymph node dissection (ALND) for pathological examination was a gold standard for the judgment of ALN metastasis in breast cancer patients. However, ALND may lead to overtreatment and increase some unnecessary postoperative complications in early-stage breast cancer patients. Sentinel lymph node biopsy (SLNB) has been recommended as a standard procedure for lymph node metastasis status which is increasingly used in clinical practice. However, it still has some problems like staining allergy and false-negative results.

There have been studies demonstrating that ultrasound radiomics might predict ALN metastasis in breast cancer in a non-invasive, safe and accurate way. In 2017, a radiomics approach to dual-model ultrasound (real-time elastography and B-mode) was proposed by Suo et al. to predict ALN metastasis in early breast cancer patients [20]. 428 radiomics features were extracted from the elastography and B-mode ultrasound images of 161 ALNs. They then used LASSO to select 35 features. The results showed that the selected features can differentiate benign from metastatic nodes with high accuracy, suggesting that dual-model ultrasound (elastography and B-mode) radiomics might help to diagnose and distinguish ALN metastasis in breast cancer patients. In 2019, Yu et al. developed a radiomics nomogram to preoperatively predict ALN metastasis in patients with early-stage invasive breast cancer (EIBC) [21]. This nomogram integrated clinical risk factors and radiomics features from ultrasound images. Four hundred and sixty-two ultrasound images of patients with EIBC were retrospectively analyzed and divided into a primary cohort $(n=300)$ and a validation cohort $(n=126)$. The results showed that the prediction efficacy of the radiomics features about ALN metastasis were moderate with AUC of 0.78 and 0.71 in the primary and validation cohorts respectively, and the radiomics nomogram had good calibration and performance for ALN detection with AUC of 0.84 and 0.81 in the primary and validation cohort respectively. It demonstrated that the radiomics nomogram has a great potential in predicting ALN metastasis. In 2020, Sun et al. compared the performance of deep CNN with radiomics analysis in predicting ALN metastasis on ultrasound and explored the role of intratumoral and peritumoral regions in forecasting ALN metastasis [22]. They retrospectively analyzed 2,395 ultrasound images from 479 cases and divided them into training cohort (343 patients, 1,715 images) and testing cohort (136 patients, 680 images). They constructed $\mathrm{CNN}$ and radiomics models based on training cohort and evaluated the models with ROC analysis based on testing cohort. Radiomics showed high accuracy with AUCs of 0.940/0.886 for combined region, 0.920/0.724 for peritumoral region, $0.913 / 0.693$ for intratumoral region in both training and testing cohorts. It suggested that ultrasound radiomics might be applied to predict ALN metastasis in breast cancer. Moreover, combining intratumoral and peritumoral regions in prediction may receive better performance.

\section{Discussion}

Radiomics is an emerging field, especially ultrasound radiomics in breast cancer. It is still in its infancy with the first relevant paper published in 2017 and only 15 papers were retrieved through PubMed and CKNI till 2020. Main applications of these literatures focused on predicting molecular subtypes $(5 / 15)$, differentiating benign from malignant tumors $(5 / 15)$, predicting the response after NACT (1/15) and estimating ALN 
metastasis (3/15) in breast cancer patients.

Breast cancer is commonly categorized into different molecular subtypes based on the expression of tumor molecular markers: estrogen receptor (ER), progesterone receptor (PR), human epidermal growth factor receptor $2\left(\mathrm{HER}^{-2-}\right)$ and Ki-67. Different molecular subtypes have different therapies and prognoses. Molecular subtype is an essential reference for providing the best patient management. In predicting molecular subtypes of breast cancer, a majority of studies focus on hormone receptors (4/5) [7-9,11], especially distinguishing Luminal-A breast cancer $\left(\mathrm{ER}^{+}, \mathrm{PR}^{+}, \mathrm{HER}^{-2-}\right)$ and $\mathrm{TNBC}\left(\mathrm{ER}^{-}\right.$, $\left.\mathrm{PR}^{-}, \mathrm{HER}^{-2-}\right)(3 / 5)[8,9,11]$. TNBC accounts for $10 \%$ $27 \%$ of whole breast cancers and presents the highest rate of recurrence and the poorest outcomes. In three studies which differentiated Luminal-A from TNBC, the extracted ultrasound radiomics features are mainly about size, shape, boundary, margin, internal echoic pattern, posterior acoustic shadow, and calcification of tumors. Among these features, margin, internal echoic pattern, posterior acoustic shadow, and calcification show statistically significant differences between Luminal-A and TNBC. According to these studies, Luminal-A have more spiculated or angular margin, hyperechoic pattern, posterior acoustic shadow and less calcification while TNBCs are more likely to show circumscribed margin, hypo- or complex internal echo, posterior acoustic enhancement and more calcification.

Furthermore, TNBC sometimes may be mistaken for fibroadenoma because of its tendency to show benign morphology on ultrasound, but treatment and prognosis of TNBC are quite different from fibroadenoma. Radiomics on ultrasound may help to distinguish them [14].

At present, studies on ultrasound radiomics are mainly based on routine ultrasound [7-11, 14-16,19,21,22]. There are also some researchers used other ultrasound imaging methods such as sonoelastography and contrast-enhanced ultrasound (CEUS) $[12,13,20,23]$. Sonoelastography can depict tissue hardness by color images. Malignant and benign tumors have different tissue hardness distributions so that their color patterns on sonoelastography are different, which is helpful for sonoelastography radiomics to differentiate benign from malignant tumors. Sonoelastography radiomics may be helpful in not only distinguishing benign from malignant breast tumors but also diagnosing and differentiating ALN metastasis in breast cancer patients [12-13,20]. Only one study based on mice suggested the feasibility of radiomics on CEUS [23].

Ultrasound radiomics in breast cancer is promising. Core needle biopsy (CNB) is the traditional way to confirm the biology characterization of breast cancer which generally only extracts and analyzes a small portion of tumor tissue, which ignores intra-tumor spatial and temporal heterogeneity. Imaging has the ability to capture tumorous heterogeneity in a non-invasive way so that it can provide a more comprehensive view of the entire tumor. Moreover, imaging is repeatable so that it can be used to monitor the progress of the disease or its response to treatment. Compared with other imaging modalities like MRI and mammography, ultrasound has the advantages of real-time, radiation-free and lowcost. Ultrasound radiomics have all the advantages of traditional ultrasound with higher sensitivity than traditional ultrasound $[8,11,19]$. Moreover, conjoint analysis of those radiomics features may provide better performance $[8,9]$.

There are several limitations of our study. Firstly, all retrieved 15 studies are retrospective study design with a relatively small simple size. Larger prospective and multicenter studies are needed to verify these preliminary results. Secondly, there are many uncertainties during procedures of image acquisition, reconstruction of calculation methods, and parameter settings of different equipment. The regions of tumors are manually delineated in some studies [15,16,20-22]. Some inevitable errors and bias from different operators may affect the reproducibility of the results. That's why efficient and standardized systems for feature extraction and data sharing need to be unified. So far as the methodology to be concerned, support vector machine were commonly used to classify the selected radiomics features [7$10,13,16,20]$, while some studies did not use the method. Besides, since different machine learning methods have different efficacy, how to choose proper method is still inconclusive $[7,13,16]$.

In conclusion, as a promising technology, the usage of ultrasound radiomics in breast cancer could assist physicians in better patient management. However, more prospective studies with higher level of evidence are required.

\section{Acknowledgments}

This work was supported by the Shenzhen 'Sanming' Project of Medicine, Health and Family Planning Commission of Shenzhen Municipality, and Shenzhen Science and Technology Innovation Committee (Grant/Award Number: SZSM201512026, SZXJ2017026 and JCYJ20170307111551748). We wish to thank Dr. Yang Hai from Jefferson Thomas Jefferson University for editing and revising the article.

\section{Conflicts of Interest}

The authors declare no conflict of interests.

\section{References}

[1] Bray F, Ferlay J, Soerjomataram I, Siegel RL, Torre LA, Jemal A. Global cancer statistics 2018: GLOBOCAN estimates of incidence 
and mortality worldwide for 36 cancers in 185 countries. CA Cancer J Clin 2018; 68: 394-424.

[2] Lambin P, Rios-Velazquez E, Leijenaar R, Carvalho S, van Stiphout RGPM, Granton P, et al. Radiomics: extracting more information from medical images using advanced feature analysis. Eur J Cancer 2012; 48: 441-6.

[3] Gillies RJ, Kinahan PE, Hricak H. Radiomics: images are more than pictures, they are data. Radiology 2016; 278: 563-77.

[4] Valdora F, Houssami N, Rossi F, Calabrese M, Tagliafico AS. Rapid review: radiomics and breast cancer. Breast Cancer Research and Treatment 2018; 169: 217-29.

[5] Crivelli P, Ledda RE, Parascandolo N, Fara A, Soro D, Conti M. A new challenge for radiologists: radiomics in breast cancer. Biomed Res Int 2018; 2018: 6120703.

[6] Kumar V, Gu Y, Basu S, Berglund A, Eschrich SA, Schabath MB, et al. Radiomics: the process and the challenges. Magn Reson Imaging 2012; 30: 1234-48.

[7] Liu T, Li J, Hu Y, Yu J, Guo Y, Wang Y, et al. Feasibility analysis of predicting expression of estrogen receptor in breast cancer based on radiomics. J Biomedical Engineering 2017; 34: 597-601.

[8] Li JW, Shi ZT, Guo Y, Hu YZ, Qiao MY, Yu JH, et al. Value of ultrasound radiomics analysis in predicting expression of hormone receptors in invasive breast carcinoma. Oncoradiology 2017; 26: 128-35.

[9] Guo Y, Hu Y, Qiao M, Wang Y, Yu J, Li J, et al. Radiomics analysis on ultrasound for prediction of biologic behavior in breast invasive ductal carcinoma. Clin Breast Cancer 2018; 18: e335-e344.

[10] Li JW, Fang Z, Zhou J, Tong YY, Shi ZT, Chang C, et al. The association between molecular biomarkers and ultrasonographic radiomics features for triple negative invasive breast carcinoma. Chin J Ultrasonography 2019; 28: 137-43.

[11] Liu BH, Jiang F, Yan N, Ji WY, Liu YH, Yao AL, et al. Clinical study on correlation between breast cancer hormone receptor expression and ultrasound radiomics. J Clin Ultras Med 2019; 21: 834-6.

[12] Zhang Q, Xiao Y, Suo J, Shi J, Yu J, Guo Y, et al. Sonoelastomics for breast tumor classification: a radiomics approach with clusteringbased feature selection on sonoelastography. Ultrasound Med Biol 2017; 43: 1058-69.

[13] Zhou Y, Xu J, Liu Q, Li C, Liu Z, Wang M, et al. A radiomics approach with CNN for shear-wave elastography breast tumor classification. IEEE Trans Biomed Eng 2018; 65: 1935-42.

[14] Lee SE, Han K, Kwak JY, Lee E, Kim EK. Radiomics of US texture features in differential diagnosis between triple-negative breast cancer and fibroadenoma. Sci Rep 2018; 8: 13546.

[15] Luo WQ, Huang QX, Huang XW, Hu HT, Zeng FQ, Wang W. Predicting breast cancer in breast imaging reporting and data system (BI-RADS) ultrasound category 4 or 5 lesions: a nomogram combining radiomics and BI-RADS. Sci Rep 2019; 9: 11921.

[16] Fleury E, Marcomini K. Performance of machine learning software to classify breast lesions using BI-RADS radiomic features on ultrasound images. Eur Radiol Exp 2019; 3: 34.

[17] Pathak M, Deo SV, Dwivedi SN, Sreenivas V, Thakur B, Julka PK, et al. Role of neoadjuvant chemotherapy in breast cancer patients: systematic review and meta-analysis. Indian J Med Paediatr Oncol 2019; 40: 48-62.

[18] Luangdilok S, Samarnthai N, Korphaisarn K. Association between pathological complete response and outcome following neoadjuvant chemotherapy in locally advanced breast cancer patients. $J$ Breast Cancer 2014; 17: 376-85.

[19] Li MY, Li B, Luo J, Liang JY, Pan FS, Zheng YL, et al. Ultrasound-based radiomics model in predicting efficacy of neoadjuvant chemotherapy in breast cancer. Chin J Med Imaging Technol 2019; 35: 1331-5.

[20] Suo J, Zhang Q, Chang W, Shi J, Yan Z, Chen M. Evaluation of axillary lymph node metastasis by using radiomics of dual-modal ultrasound composed of elastography and B-mode. Chin J Med Instrument 2017; 41: 313-316.

[21] Yu FH, Wang JX, Ye XH, Deng J, Hang J, Yang B. Ultrasound-based radiomics nomogram: a potential biomarker to predict axillary lymph node metastasis in early-stage invasive breast cancer. Eur J Radiol 2019; 119: 108658.

[22] Sun Q, Lin X, Zhao Y, Li L, Yan K, Liang D, et al. Deep learning vs. radiomics for predicting axillary lymph node metastasis of breast cancer using ultrasound images: don't forget the peritumoral region. Front Oncol 2020; 10: 53.

[23] Theek B, Opacic T, Magnuska Z, Lammers T, Kiessling F. Radiomic analysis of contrast-enhanced ultrasound data. Sci Rep 2018; 8: 11359 . 